\title{
The GRONORUN 2 study: effectiveness of a preconditioning program on preventing running related injuries in novice runners. The design of a randomized controlled trial
}

\author{
Steef W Bredeweg ${ }^{1,2^{*}+}$, Sjouke Zijlstra ${ }^{1,2+}$, Ida Buist ${ }^{1,2+}$
}

\begin{abstract}
Background: Distance running is a popular recreational exercise. It is a beneficial activity for health and well being. However, running may also cause injuries, especially of the lower extremities. In literature there is no agreement what intrinsic and extrinsic factors cause running related injuries (RRIs). In theory, most RRIs are elicited by training errors, this too much, too soon. In a preconditioning program runners can adapt more gradually to the high mechanical loads of running and will be less susceptible to RRIs. In this study the effectiveness of a 4-week preconditioning program on the incidence of RRIs in novice runners prior to a training program will be studied.

Methods/Design: The GRONORUN 2 (Groningen Novice Running) study is a two arm randomized controlled trial studying the effect of a 4-week preconditioning (PRECON) program in a group of novice runners. All participants wanted to train for the recreational Groningen 4-Mile running event. The PRECON group started a 4-week preconditioning program with walking and hopping exercises 4 weeks before the start of the training program. The control (CON) and PRECON group started a frequently used 9-week training program in preparation for the Groningen 4-Mile running event.

During the follow up period participants registered their running exposure, other sporting activities and running related injuries in an Internet based running log. The primary outcome measure was the number of RRIs. RRI was defined as a musculoskeletal ailment or complaint of the lower extremities or back causing a restriction on running for at least three training sessions.
\end{abstract}

Discussion: The GRONORUN 2 study will add important information to the existing running science. The concept of preconditioning is easy to implement in existing training programs and will hopefully prevent RRIs especially in novice runners.

Trial registration: The Netherlands National Trial Register NTR1906. The NTR is part of the WHO Primary Registries.

\section{Background}

Running is a popular activity and can be practised everywhere. The health benefits are substantial but runners get injured regularly. The incidence of running related injuries (RRIs) is high. Various studies in different populations reported rates of RRIs ranging from $19-79 \%$ [1-9]. RRIs are often located in the lower

\footnotetext{
* Correspondence: s.w.bredeweg@sport.umcg.nl

† Contributed equally

'University Center of Sport, Exercise and Health, University Medical Center Groningen, Hanzeplein 1, 9700 RB Groningen, The Netherlands

Full list of author information is available at the end of the article
}

extremities with knee and lower leg mostly affected $[3,6,7,10-12]$. There is no agreement on the cause of RRIs. In the current literature, possible intrinsic and extrinsic risk factors are identified, but there is still no exact cause for an RRI. Van Mechelen [13] and van Gent et al [9] proposed risk factors that have been significantly related to RRI; excessive weekly running distance, previous injury, lack of running experience and competitive running.

Clinical studies showed that over $60 \%$ of RRIs could be attributed to training errors [14]. Hreljac [14] stated 
that all overuse running injuries are the result of training errors. From this point of view a RRI is a disturbance between the external load applied to the body and the injury threshold of a biological structure of the body. In this dose-response relationship there are four components applicable to the novice runner [14]. The first component is the current status of the musculoskeletal system of the novice runner. The second component is the type of applied stress (i.e. running). Thirdly, the frequency, intensity and duration of the applied stress (i.e. running) and finally, the adaptation and recovery times between running sessions are major determinants of this dose relationship.

Under normal circumstances the musculoskeletal system adapts to the level of stress placed upon it [14-16]. When an optimal level of stress is applied to the musculoskeletal system, along with an adequate recovery time, the musculoskeletal system will increase in strength. On the other hand, when the applied stress is too high or the recovery time is too short the tissue of the musculoskeletal system will be weakened and the likelihood of sustaining a subsequent overuse injury is high $[14,16,17]$. Mechanical load (i.e. running) applied to the human body can cause a physiological or pathological adaptation to this mechanical loading, resulting in respectively a training effect or overuse injury [17].

The musculoskeletal system of the novice runner is normally not adapted to the repetitive and relatively high impact forces of running because novice runners are frequently physically inactive before they start to run $[10,18]$. In most regular running programs for novice runners the biomechanical load is high from the start of the program in terms of frequency, intensity and duration.

The first GRONORUN study $[7,19]$ showed that previous sports participation without axial loading was an important predictor for RRIs in novice runners. From this knowledge a strategy can be chosen to strengthen the lower extremities to achieve a positive physiological adaptation of the musculoskeletal system before starting a training program for novice runners. The applied external load of this so called preconditioning program will stress the lower extremities and as a result the lower extremities will positively adapt to the applied stress. In this way there is a stepwise transition of biomechanical load which makes it easier for the musculoskeletal system of the lower extremities to withstand the demands of running. Other studies in athletes and military populations [20-23,23] showed a positive effect of a preconditioning program on the incidence of sports and overuse injuries in different populations.

In a preconditioning program for running, the program needs to load the musculoskeletal system in a sport-specific way. Therefore, in this randomized controlled trial a preconditioning program with walking and hopping prior to the training program for novice runners will be studied. We hypothesize that the novice runner can adapt more gradually to the external impact forces of running with a preconditioning program prior to the training program and will be less susceptible to RRIs.

\section{Methods/design}

The GROningen NOvice RUNing 2 (GRONORUN 2) study is a randomized controlled trial with a 13-week follow-up. Participants were randomized into two groups: an active control (CON) group and an intervention (PRECON) group. The PRECON group will receive a 4-week preconditioning program prior to the start of the training program. Recruitment of participants for the GRONORUN 2 study took place in the period April June 2008 and data collection started in July 2008. The study design, procedures and informed consent procedure were approved by the Medical Ethics Committee of the University Medical Center Groningen (No. 2007.217). All participants provided written informed consent. Guidelines were followed according to the Consort Statement [24].

\section{Study population}

In the period April - June 2008, participants who were willing to start a "beginners 9-week program" in preparation for the Groningen 4-Mile running event were recruited with advertisements in local media in the northern part of the Netherlands. For this study participants were not obliged to participate in the Groningen 4-Mile running event. The Groningen 4-Mile running event is a popular annual recreational running event that takes place in October. After initial registration, potential participants were sent written information about the study along with a baseline questionnaire and invitation for an initial interview in the Center for Sports Medicine at the University Medical Center Groningen (UMCG), The Netherlands.

\section{Inclusion \& exclusion criteria}

Healthy subjects between 18 and 65 years of age who had no injury of lower extremities or lower back in the last three months prior to inclusion, who had not been running on a regular basis in the previous twelve months who were willing to start a beginners program were eligible for inclusion in the study. Potential participants were excluded to the study if there were absolute contraindications for vigorous physical activities according to the American College of Sports Medicine [25] or in case of unwillingness to keep a running log.

\section{Sample size}

A power calculation was carried out for the main outcome variable, i.e. running related injury (RRI), using a 
logistic rank survival power analysis. As stated before, the incidence of RRIs varies between 19-79\%.

A reduction of $25 \%$ on the incidence of RRIs in the PRECON group is considered clinically significant and relevant. The expected incidence of RRIs is $40 \%[4,10]$. With a hypothesized $25 \%$ reduction of RRIs in the PRECON group compared to the control group, a total of 360 runners $(2 \times 180)$ is needed for a power of $80 \%$ and an alpha of 0.05 . Assuming an attrition of $15 \%$ in the intervention period and follow up period, a total of 414 $(2 \times 207)$ novice runners are needed to detect an effect of the PRECON intervention.

\section{Baseline questionnaires}

All participants filled in an online questionnaire before baseline measurements were taken. In case potential participants had no access to the internet a questionnaire was sent by mail. Demographic and anthropometric variables that were collected were age, gender, body weight and length. Conditions related to risk factors for cardiovascular diseases were assessed using a series of questions according to the American College of Sports Medicine [25]. Past musculoskeletal complaints of the lower extremities and back were assessed by questions on the anatomical site and the number of days lost to work and/or sporting activities. When a musculoskeletal complaint was caused by a sporting activity it was registered as a previous sports injury. When the musculoskeletal complaint was caused by running in the past it was registered as a previous running injury. Sports participation was measured by asking whether someone was participating in sports in the past twelve months (yes/no), type of sport and mean hours of sport participation per sport a week. Furthermore a question on running experience in the past ("did you ever structurally run before") was added to assess the novelty to running.

After receiving the complete questionnaire potential participants were invited for an initial interview by an experienced sports physician at the Sportsmedicine Center of the University Medical Center Groningen. The purpose of the initial interview was to screen for cardiovascular diseases and abnormalities of lower limb and to ensure that the participants were eligible and were adequately informed about the study before signing informed consent for the GRONORUN 2 study.

\section{Baseline orthopaedic measurements}

Hip function was measured by using a universal goniometer with arm length $30 \mathrm{~cm}$ from axis to tip. The internal and external range of motion of the hip was assessed with the participant supine and the tested hip and knee flexed to $90^{\circ}$. Knee flexion and extension ranges of motion were assessed with the participant in supine position. The goniometer was placed on the lateral aspect of the knee, with the axis of the goniometer in line with the greater trochanter and the lateral malleolus. Ankle plantar flexion and dorsi flexion were measured both with the knee fully extended and flexed to $90^{\circ}$. One arm of the goniometer was aligned with the fibular bone and the other with the plantar surface of the foot. Furthermore, the navicular drop was assessed by measuring the change in the height of the navicular tuberosity between a participant sitting with the subtalar joint in neutral position and standing, weight bearing with the subtalar joint in relaxed stance, as described by Brody [26]. The navicular drop is a valid method to indicate the amount of foot pronation [27]. Intratester and intertester reliability of this technique is ranging from .73 to .96 [28]. Measurements were made twice for each foot, with results being averaged. These measurements were identical to the GRONORUN 1 study [19].

\section{Randomization}

After baseline measurements and informed consent, participants were randomly assigned to the $\mathrm{CON}$ or the PRECON training program.

To ensure that both groups were equal in terms of injury risk, a stratified randomization was performed based on three variables; current sporting activities, previous injuries and gender. Based on current sporting activities, there were three categories of novice runners. The first category consisted of novice runners who already were participating in a sport in which axial load i.e. running, walking or jumping, was integrated. The second category was formed by novice runners who already were participating in sporting activities without axial load, like swimming and cycling. The third and last category was formed by novice runners who did not participate in any sporting activities at baseline measurements.

In a study by Macera [1], a $74 \%$ increased risk was found in runners with a positive history of previous injuries. In this study, previous musculoskeletal complaints with an impact of activities of daily life, work or sporting activities were defined as a previous injury. Since it is not clear whether the high rate of re-injury is caused by incomplete healing of a previous injury or a biomechanical problem, a differentiation in time is made. A distinction can be made between no previous injury, injuries sustained in the last 12 months before baseline measurements and injuries sustained more than 12 months before baseline measurements. The participants were also stratified for gender because men and women differ in incidence of RRIs and localisation of these injuries $[6,9]$. In total eighteen strata were formed by gender, previous injury (no injuries, injury 3 till 12 months ago and injuries longer then 12 months ago) 
and sporting activities (no, with axial load and without axial load). From each stratum, participants were randomly allocated to intervention or control group by drawing a sealed opaque envelope. Each stratum box contained equal numbers of control and intervention envelopes.

\section{Participant flow}

The study design and participants flow are shown in Figure 1. A total of 500 people were interested to participate in the GRONORUN 2 study and responded to the call for novice runners. To all of those who reacted on the advertisements, an information brochure in which the study protocol was clearly described, a baseline questionnaire and an appointment at the UMCG was given. Forty four did not confirm their appointment for the initial interview nor filled in the baseline questionnaire. Of those who confirmed the appointment for the initial interview and filled in the questionnaire $(\mathrm{n}=$ 456), four failed to attend the initial interview. Eventually, of the 452 persons who visited the UMCG for an initial interview, 20 were excluded. Reasons for exclusion were: already participating in running $(n=11)$, musculoskeletal injury of lower extremities or back at baseline $(n=6)$ and contraindications for vigorous physical activity $(\mathrm{n}=3)$. After baseline measurements and stratification, 432 participants were randomly assigned to the intervention group $(\mathrm{n}=211)$ and to the control group $(\mathrm{n}=221)$.

\section{Movie: Correct hopping technique Training program}

4-week preconditioning program The PRECON group received a 4-week individual preconditioning training program (Table 1 ). This program gradually increases biomechanical load on the lower extremities with walking and hopping sessions. Participants were instructed to walk briskly on their running shoes three times a week. During two of the three walking sessions per week, participants carried out hopping exercises. After every five minutes of walking a session of hopping was carried out. In approximately half an hour six sessions of hopping were carried out. The number of hops as well as the weekly walking distance increased gradually. The PRECON group received verbal information about the correct hopping technique at the initial interview and there was a video instruction on the personalized environment of the internet based training log of the PRECON group.

The correct technique of hopping in place was a relaxed standing position with a distance of approximately $30 \mathrm{~cm}$ between the left and right foot with both hands in the sides. Then small jumps in place were performed with the forefeet almost keeping contact with the ground (Additional file 1). During the 4 weeks of the preconditioning program of the PRECON group the $\mathrm{CON}$ group was instructed to do their normal exercise routine and sporting activities if applicable.

In the first training week, in which both groups are starting to run, the theoretical extra biomechanical load of running $(10 \mathrm{~km} / \mathrm{h}$; total $30 \mathrm{~min}$, impact 2.0 bodyweight (BW) per landing) and walking $(5 \mathrm{~km} / \mathrm{h}$, total $30 \mathrm{~min}$, impact $1.0 \mathrm{BW}$ per landing) is approximately 12,500 extra BW. Walking is a low cyclic impact force activity and hopping is a high cyclic impact force activity. Both activities stress the body in a cyclic way, especially the lower extremities, so that the body has to positively adapt to the biomechanical stimuli.

In the first week of the PRECON program the extra biomechanical load of walking $(5 \mathrm{~km} / \mathrm{h}$, total $90 \mathrm{~min}$, impact 1.0 BW per landing) and hopping (660 hops, impact per hop $3.5 \mathrm{BW}$ per landing) was approximately 9810 extra BW. In week 2 there is 11480 extra BW, in week $313150 \mathrm{BW}$ and in week $413570 \mathrm{BW}$ (Figure 2).

The 9-week training program Nine weeks before the Groningen 4-Mile running event all participants were instructed to start their 9-week training program (Table 2). Participants of the CON and PRECON group received the same general written and oral information on intensity of running and on warming up and cooling down. Participants were instructed to walk briskly for 5 minutes as a warm up, and 5 minutes as cool down. Given that the best available evidence indicates that stretching before or after exercise does not prevent muscle soreness or injury [29], participants were instructed not to perform stretching exercises before, during or after the training sessions.

The frequency of running was equal in both groups. Each training week except the last week i.e. the week of the Groningen 4-Mile running event, consisted of three training sessions represented by a combination of running and walking. Participants were encouraged to run on Monday, Wednesday and Saturday or on Tuesday, Thursday and Sunday. Runners were advised to run at a comfortable pace at which they could converse without breathlessness. Both groups trained individually, without a trainer on a self-chosen course.

\section{Outcome measures}

The primary outcome of the GRONORUN trial was the number of RRIs in both groups. A runner could only have one RRI. Definition of a RRI in this trial was; running related musculoskeletal ailment of the lower extremities or back, causing a restriction of running for at least one week, i.e. three consecutive training sessions.

Information on RRIs and exposure data was collected using an internet based running log. Each of the participants received a study number and a password to enter a personal environment of the web based training log. 


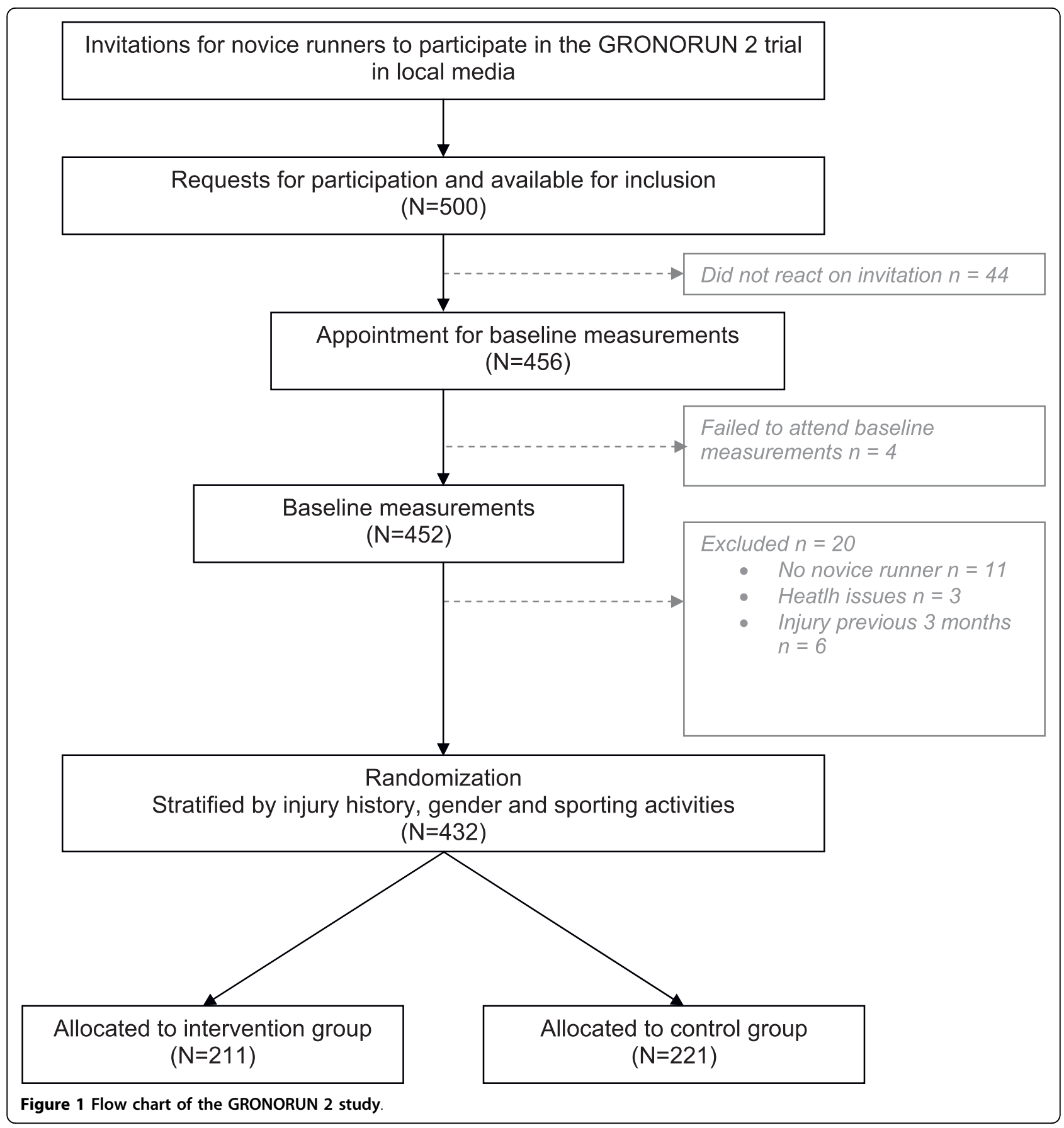

After each training week participants had to fill in their running activities, other sport activities and injuries.

Per training session the total minutes of running, total minutes of walking and injuries were registered. Data on injuries were collected by registering anatomical site of the body and severity of pain. Severity of pain was subdivided in pain without limitation (no RRI), pain that caused a restriction of running (scored as an RRI) and pain which made running impossible RRI (scored as an
RRI). In case of skipping a training session, the reason (RRI, other injury, motivation, illness or remaining reason) for it was asked. When a "running related injury" was the reason for not training, information on anatomical site and severity was asked. To point out the anatomical site of an injury, a picture of the lower body was shown after reporting a RRI. By clicking on the anatomical site of the RRI, the same spot was appointed in red. When participants did not enter their digital training log 
Table 1 The 4-week preconditioning (PRECON) program with walking and hopping

\begin{tabular}{|c|c|c|c|c|c|c|c|c|c|}
\hline & \multicolumn{3}{|c|}{ PRECON training 1} & \multicolumn{3}{|c|}{ PRECON training 2} & \multirow{2}{*}{$\begin{array}{c}\text { PRECON training } 3 \\
\begin{array}{c}\text { Walk } \\
\text { (min) }\end{array} \\
\end{array}$} & \multicolumn{2}{|c|}{ Total } \\
\hline & $\begin{array}{l}\text { Walk } \\
\text { (min) }\end{array}$ & Hop & (rep.) & $\begin{array}{l}\text { Walk } \\
\text { (min) }\end{array}$ & Hop & (rep.) & & $\begin{array}{l}\text { Walk } \\
\text { (min) }\end{array}$ & Hop \\
\hline week 1 & 5 & 50 & (6) & 5 & 60 & (6) & 30 & $\begin{array}{c}90 \\
{[7500 \mathrm{BW}]}\end{array}$ & $\begin{array}{c}660 \\
{[2310 \mathrm{BW}]}\end{array}$ \\
\hline week 2 & 5 & 60 & (6) & 5 & 70 & (6) & 45 & $\begin{array}{c}105 \\
{[8750 \mathrm{BW}]}\end{array}$ & $\begin{array}{c}780 \\
{[2730 \mathrm{BW}]}\end{array}$ \\
\hline week 3 & 5 & 70 & (6) & 5 & 80 & (6) & 60 & $\begin{array}{c}120 \\
{[10000 \mathrm{BW}]}\end{array}$ & $\begin{array}{c}900 \\
{[3150 \mathrm{BW}]}\end{array}$ \\
\hline week 4 & 5 & 80 & (6) & 5 & 90 & (6) & 60 & $\begin{array}{c}120 \\
{[10000 \mathrm{BW}]}\end{array}$ & $\begin{array}{c}1020 \\
{[3570 \mathrm{BW}]}\end{array}$ \\
\hline week 5 & Start of th & ek t & prog & & & & & & \\
\hline
\end{tabular}

The content of the PRECON training program is expressed in minutes of walking (Walk) and the number of hopping in place (Hop) and the amount of repetitions (rep.) of walking and hopping. The right column contains total minutes of walking and total performed hops each week. In the last two columns the additional load of walking and hopping each week is expressed between brackets [] in bodyweight (BW)

after one week, a reminder was send by email automatically. In case of not having access to the Internet, all participants had also a hard copy of the running log.

\section{Statistical analyses}

To evaluate the success of the randomization, baseline characteristics of participants in the CON and PRECON group were compared using 2-tailed t-tests for normally distributed continuous variables. The $\chi^{2}$ statistic was used for discrete variables. To evaluate the effect of the PRECON program on the number of injured runners in both groups, a $\chi^{2}$-test was used. The log-rank test is used to compare the Kaplan-Meier curves of the injured runners of the PRECON group and the CON group, analyzing the difference between these two groups in the probability of an RRI at any point in time. All analyses were performed following the "intention to treat" principle. Differences were considered statistically significant at $\mathrm{P}<.05$. All analyses were performed using SPSS version 16.0 (SPSS Inc, Chicago, Ill).

\section{Discussion}

To study the population of novice runners it is important because the main reason for discontinuation (drop out) of a running program is injury [18]. Negative experiences, caused by an injury that occurs while training for a running event, have the potential to significantly affect the future physical activity of each

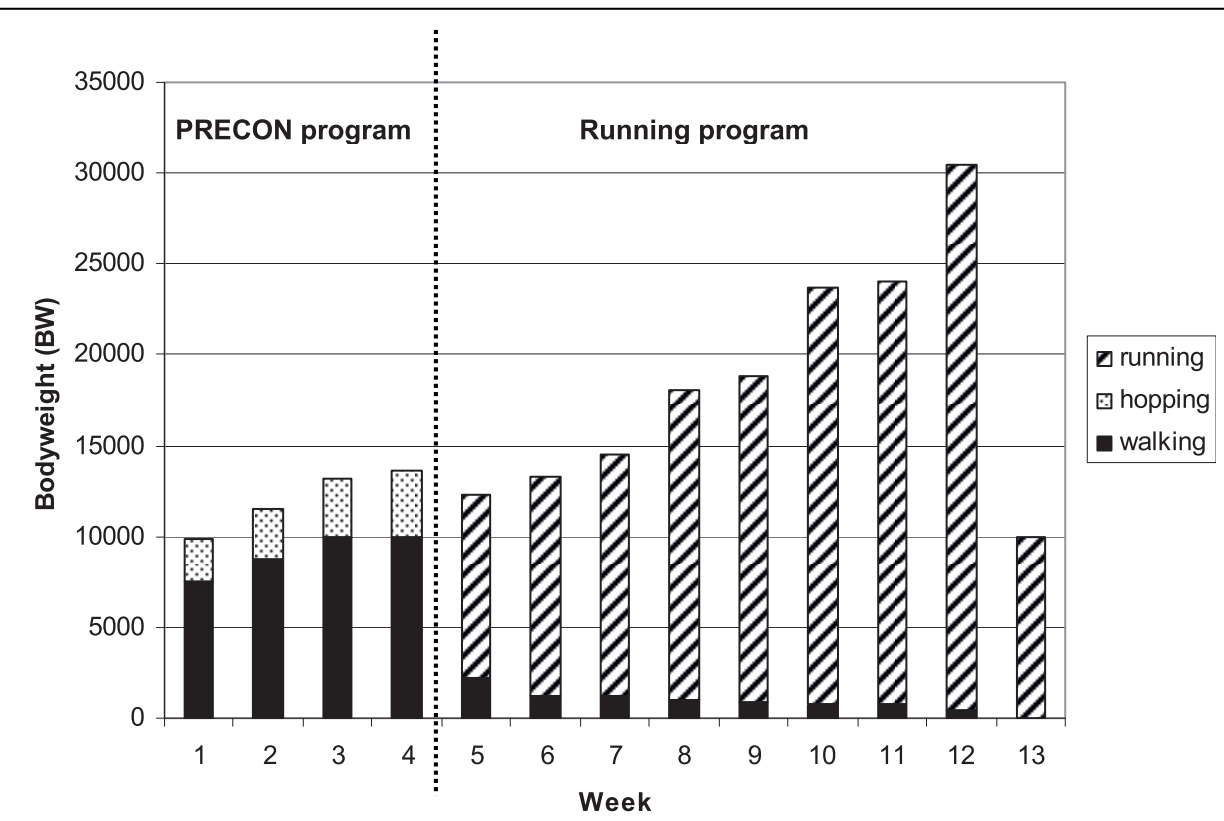

Figure 2 Biomechanical load for the PRECON (week 1-13) and the CON (week 5-13) group. The load of walking, hopping and running is expressed in bodyweight (BW) per week. Week 1-4 was the intervention period of the PRECON group. From week 5 both groups followed the same training program. 
Table 2 The 9-week training program for both groups

\begin{tabular}{|c|c|c|c|c|c|c|c|c|c|c|c|}
\hline \multirow{2}{*}{\multicolumn{2}{|c|}{ run }} & \multicolumn{3}{|c|}{ Training 1} & \multicolumn{3}{|c|}{ Training 2} & \multicolumn{2}{|c|}{ Training 3} & \multicolumn{2}{|c|}{ Total } \\
\hline & & walk & (rep.) & run & Walk & (rep.) & run & walk & (rep.) & Run & walk \\
\hline week 1 & 1 & 1 & (10) & 1 & 1 & (10) & 1 & 1 & (10) & 30 & 27 \\
\hline week 2 & 2 & 1 & (6) & 2 & 1 & (6) & 2 & 1 & (6) & 36 & 15 \\
\hline week 3 & 4 & 2 & (3) & 4 & 2 & (4) & 4 & 2 & (3) & 40 & 14 \\
\hline week 4 & 6 & 2 & (3) & 5 & 2 & (3) & 6 & 2 & (3) & 51 & 12 \\
\hline week 5 & 6 & 2 & (3) & 9 & 2 & (2) & 6 & 2 & (3) & 54 & 10 \\
\hline week 6 & 8 & 2 & (3) & 15 & 0 & (1) & 15 & 5 & (2) & 69 & 9 \\
\hline week 7 & 10 & 2 & (2) & 15 & 5 & (2) & 10 & 2 & (2) & 70 & 9 \\
\hline week 8 & 30 & 0 & (1) & 15 & 5 & (2) & 30 & 0 & (1) & 90 & 5 \\
\hline week 9 & 30 & 0 & (1) & & & & & ningen & -Mile event & 30 & 0 \\
\hline
\end{tabular}

The content of each training session is expressed in minutes of running (run), minutes of walking between the running sessions (walk) and number of repetitions (rep.). The last two columns contains the total minutes of running and walking for each week.

individual. It is also known that (fear of) sustaining an injury is associated with failure to start and maintain a physically active lifestyle [30].

As stated by Yeung [31] there is a need for more well controlled trials to shed light on possible interventions for the prevention of lower limb injuries in runners. Current studies on the effect of interventions for preventing running injuries in recreational runners are scarce. The GRONORUN 1 study [7] showed no effect of a more gradual training program in the novice recreational runners.

In preventive medicine it is important to develop interventions based on the understanding of the etiology and mechanisms of injury and the preventive intervention has to be acceptable, practical and adopted by athletes and sport bodies so that the implementation of the intervention can be successful [32]. The proposed intervention in this RCT is practical, easy to do and therefore has a good chance for success in terms of compliance, efficacy and effectiveness.

Results of this GRONORUN 2 study can be implemented in the existing training program for novice runners and the new preconditioning training program can be implemented on a regional, national and international level. In this way, a more scientific based training program for novice runners can be developed and novice runners will feel safer in starting a running program.

With this study there is also a unique opportunity to start more clinical and preventive studies on overuse running related injuries. The newly gathered information will be transferred into new clinical and preventive studies in the future.

\section{Additional material}

Additional file 1: Suppl1.

\section{Acknowledgements}

The authors thank Hans Zwerver, Bram Bessem, Steven Doeven, Stijn de Bruijn en Marianne Roossien for their help at the inclusion of the participants for this study. All were funded by the University Medical Center Groningen.

SZ holds a research fellowship funded by the Netherlands Organisation for Health Research and development (ZonMW). SWB and IB are funded by the Netherlands Organisation for Health Research and development (ZonMW) and the University Medical Center Groningen

This study was funded by the Netherlands Organisation for Health Research and development (ZonMW), grant number 75020009. The funding body ZonMW, did not play a role in the study design, and in the collection, analysis or interpretation of these data.

\section{Author details}

${ }^{1}$ University Center of Sport, Exercise and Health, University Medical Center Groningen, Hanzeplein 1, 9700 RB Groningen, The Netherlands. ${ }^{2}$ Center for Sports Medicine, University Medical Center Groningen, Hanzeplein 1, 9700 RB Groningen, The Netherlands.

\section{Authors' contributions}

SWB conceived of the idea, obtained funding for the study and developed the intervention. SWB and IB developed the design of this trial and recruited participants. SWB is the study investigator and wrote the article, SZ was responsible for data acquisition. IB was co applicant of the grant.

All authors read and approved the final manuscript.

\section{Competing interests}

The authors declare that they have no competing interests.

Received: 15 June 2010 Accepted: 1 September 2010

Published: 1 September 2010

\section{References}

1. Macera CA, Pate RR, Powell KE, Jackson KL, Kendrick JS, Craven TE: Predicting lower-extremity injuries among habitual runners. Arch Intern Med 1989, 149:2565-2568.

2. Lun V, Meeuwisse WH, Stergiou P, Stefanyshyn D: Relation between running injury and static lower limb alignment in recreational runners. Br J Sports Med 2004, 38:576-580.

3. Walter SD, Hart LE, McIntosh JM, Sutton JR: The Ontario cohort study of running-related injuries. Arch Intern Med 1989, 149:2561-2564.

4. Bovens AMP, Janssen GME, Vermeer HGW, Hoeberigs JH, Janssen MPE, Verstappen FTJ: Occurrence of Running Injuries in Adults Following A Supervised Training-Program. International Journal of Sports Medicine 1989, 10:S186-S190.

5. Lysholm J, Wiklander J: Injuries in Runners. American Journal of Sports Medicine 1987, 15:168-171. 
6. Buist I, Bredeweg SW, Bessem B, Van MW, Lemmink KA, Diercks RL: Incidence and risk factors of Running-Related Injuries during preparation for a four-mile recreational running event. Br J Sports Med 2010, 44:598-604.

7. Buist I, Bredeweg SW, Van MW, Lemmink KA, Pepping GJ, Diercks RL: No effect of a graded training program on the number of running-related injuries in novice runners: a randomized controlled trial. Am J Sports Med 2008, 36:33-39.

8. Van MM, Kolkman J, Van OJ, Bierma-Zeinstra SM, Koes BW: Risk factors for lower extremity injuries among male marathon runners. Scand J Med Sci Sports 2008, 18:691-697.

9. van Gent RN, Siem D, van Middelkoop M, van Os AG, Bierma-Zeinstra SMA, Koes BW: Incidence and determinants of lower extremity running injuries in long distance runners: a systematic review. British Journal of Sports Medicine 2007, 41:469-480.

10. Taunton JE, Ryan MB, Clement DB, McKenzie DC, Lloyd-Smith DR, Zumbo BD: A prospective study of running injuries: the Vancouver Sun Run "In Training" clinics. Br J Sports Med 2003, 37:239-244.

11. van Middelkoop M, Kolkman J, Van Ochten J, Bierma-Zeinstra SMA, Koes B Prevalence and incidence of lower extremity injuries in male marathon runners. Scandinavian Journal of Medicine \& Science in Sports 2008, 18:140-144.

12. James SL, Bates BT, Ostering LR: Injury to runners. American Journal of Sports Medicine 1978, 6:40-50.

13. van Mechelen W: Running injuries. A review of the epidemiological literature. Sports Med 1992, 14:320-335.

14. Hreljac A: Impact and overuse injuries in runners. Med Sci Sports Exerc 2004, 36:845-849.

15. Kjaer M: The secrets of matrix mechanical loading. Scand J Med Sci Sports 2009, 19:455-456.

16. Kjaer M: Role of extracellular matrix in adaptation of tendon and skeletal muscle to mechanical loading. Physiol Rev 2004, 84:649-698.

17. Kjaer M, Magnusson P, Krogsgaard M, Boysen Moller J, Olesen J, Heinemeier K, Hansen M, Haraldsson B, Koskinen S, Esmarck B, Langberg H: Extracellular matrix adaptation of tendon and skeletal muscle to exercise. J Anat 2006, 208:445-450.

18. Chorley JN, Cianca JC, Divine JG, Hew TD: Baseline injury risk factors for runners starting a marathon training program. Clin J Sport Med 2002, 12:18-23.

19. Buist I, Bredeweg SW, Lemmink KA, Pepping GJ, Zwerver J, van Mechelen W, Diercks RL: The GRONORUN study: is a graded training program for novice runners effective in preventing running related injuries? Design of a Randomized Controlled Trial. BMC Musculoskelet Disord 2007, 8:24.

20. Gabbe BJ, Finch CF, Wajswelner H, Bennell KL: Predictors of lower extremity injuries at the community level of Australian football. Clin $J$ Sport Med 2004, 14:56-63.

21. Knapik JJ, Darakjy S, Hauret KG, Canada S, Scott S, Rieger W, Marin R, Jones $\mathrm{BH}$ : Increasing the physical fitness of low-fit recruits before basic combat training: An evaluation of fitness, injuries, and training outcomes. Military Medicine 2006, 171:45-54.

22. Knapik JJ, Swedler DI, Grier TL, Hauret KG, Bullock SH, Williams KW, Darakjy SS, Lester ME, Tobler SK, Jones BH: Injury reduction effectiveness of selecting running shoes based on plantar shape. J Strength Cond Res 2009, 23:685-697.

23. Askling C, Karlsson J, Thorstensson A: Hamstring injury occurrence in elite soccer players after preseason strength training with eccentric overload. Scand J Med Sci Sports 2003, 13:244-250.

24. Moher D, Schulz KF, Altman DG: The CONSORT statement: revised recommendations for improving the quality of reports of parallel-group randomised trials. Lancet 2001, 357:1191-1194.

25. Balady GJ, Chaitman B, Driscoll D, Foster C, Froelicher E, Gordon N, Pate R, Rippe J, Bazzarre T: Recommendations for cardiovascular screening, staffing, and emergency policies at health/fitness facilities. Circulation 1998, 97:2283-2293.

26. Brody DM: Techniques in the Evaluation and Treatment of the Injured Runner. Orthopedic Clinics of North America 1982, 13:541-558.

27. Mueller MJ, Host JV, Norton BJ: Navicular Drop As A Composite Measure of Excessive Pronation. Journal of the American Podiatric Medical Association 1993, 83:198-202.
28. Sell KE, Verity TM, Worrell TW, Pease BJ, Wigglesworth J: 2 Measurement Techniques for Assessing Subtalar Joint Position - A Reliability Study. Journal of Orthopaedic \& Sports Physical Therapy 1994, 19:162-167.

29. Herbert RD, Gabriel M: Effects of stretching before and after exercising on muscle soreness and risk of injury: systematic review. British Medical Journal 2002, 325:468-470A.

30. Sallis JF, Hovell MF, Hofstetter CR: Predictors of Adoption and Maintenance of Vigorous Physical-Activity in Men and Women. Preventive Medicine 1992, 21:237-251.

31. Yeung EW, Yeung SS: A systematic review of interventions to prevent lower limb soft tissue running injuries. Br J Sports Med 2001, 35:383-389.

32. Finch $C$ : A new framework for research leading to sports injury prevention. J Sci Med Sport 2006, 9:3-9.

\section{Pre-publication history}

The pre-publication history for this paper can be accessed here: http://www.biomedcentral.com/1471-2474/11/196/prepub

doi:10.1186/1471-2474-11-196

Cite this article as: Bredeweg et al:: The GRONORUN 2 study: effectiveness of a preconditioning program on preventing running related injuries in novice runners. The design of a randomized controlled trial. BMC Musculoskeletal Disorders 2010 11:196.

\section{Submit your next manuscript to BioMed Central and take full advantage of:}

- Convenient online submission

- Thorough peer review

- No space constraints or color figure charges

- Immediate publication on acceptance

- Inclusion in PubMed, CAS, Scopus and Google Scholar

- Research which is freely available for redistribution

Submit your manuscript at www.biomedcentral.com/submit
C Biomed Central 